Revue de l'Institut des langues et cultures

d'Europe, Amérique, Afrique, Asie et Australie

28 | 2017

Passages, ancrage dans la littérature de voyage

\title{
Les traditions culinaires : ancrage ou libération culturelle? Une étude des memoires culinaires
}

Culinary Traditions: Anchor or Cultural Release? A Study of Culinary Memoirs

\section{Virginia Allen-Terry Sherman}

\section{OpenEdition}

Journals

Édition électronique

URL : http://journals.openedition.org/ilcea/4165

DOI : 10.4000/ilcea.4165

ISSN : 2101-0609

\section{Éditeur}

UGA Éditions/Université Grenoble Alpes

Édition imprimée

ISBN : 978-2-84310-374-2

ISSN : 1639-6073

Référence électronique

Virginia Allen-Terry Sherman, « Les traditions culinaires : ancrage ou libération culturelle? Une étude des memoires culinaires », ILCEA [En ligne], 28 | 2017, mis en ligne le 06 mars 2017, consulté le 23 avril 2019. URL : http://journals.openedition.org/ilcea/4165 ; DOI : 10.4000/ilcea.4165

Ce document a été généré automatiquement le 23 avril 2019

(C) ILCEA 


\title{
Les traditions culinaires : ancrage ou libération culturelle? Une étude des memoires culinaires
}

Culinary Traditions: Anchor or Cultural Release? A Study of Culinary Memoirs

\author{
Virginia Allen-Terry Sherman
}

1 Les récits de voyage relatent des périples articulés autour du déplacement ou du déracinement de personnages et qui permettent la découverte de nouveaux points d'ancrage et de nouvelles traditions. Celles-ci peuvent entraver le voyageur comme autant de symboles d'inertie et de contraintes sociales. Cependant dans un genre nouveau tel que le récit autobiographique de voyages, psychiques ou physiques ${ }^{1}$, marqué par un idiome culinaire dominant qui préserve et transmet la notion d'ethnicité, les traditions peuvent représenter une force positive à respecter émanant des familles protectrices et des communautés d'appartenance, tout en offrant la possibilité de retrouver une part de liberté et d'intégrité personnelle. La dimension autobiographique prend alors une place importante car l'ancrage, déterminé par des repères prescrits par la communauté, se définit dans l'intimité de l'histoire personnelle. Nous prendrons l'exemple de deux histoires personnelles, celle de Shoba Narayan dans Monsoon Diary (2003) et de M. F. K. Fisher dans The Gastronomical Me (1989) pour explorer comment deux femmes, à soixante ans d'écart, utilisent la nourriture comme moyen de gagner une certaine independence et (re)négocier leur identité de migrantes entre les pratiques feminines traditionelles et modernes propres à leur époque et à leur culture.

\section{Les mémoires culinaires, un genre littéraire émergent}

2 Fruits hybrides de la littérature gastronomique, les mémoires culinaires sont essentiellement contemporains, anglophones et d'auteurs souvent d'origine diasporique. Ils offrent un cadre littéraire pour les peuples dispersés en quête d'identité et préoccupés de raviver des souvenirs, de trouver un équilibre et de se reconstruire. Pour James Olney, 
l'autobiographie extériorise l'impulsion enfouie au plus profond de soi de trouver son intégralité, besoin également motivé par le traumatisme de l'aliénation, au cœur de la conscience diasporique.

Le genre autobiographique, au sens large, exprime notre désir d'imposer de l'ordre dans nos vies, tout en soulageant l'anxiété qui accompagne la désintégration de soi, conséquence souvent inévitable de l'exil (Anderson, 2001 : 4-5). Le lecteur recherche son intégrité dans l'histoire des autres, en même temps que les auteurs tentent de créer de l'ordre dans la remémoration. En tant que "manuels pratiques", le sous-genre des mémoires culinaires associent l'anamnèse à l'acte de création à travers des recettes qui invitent le lecteur à cuisiner. L'acte créatif, initié par le narrateur des récits autobiographiques culinaires (appelés également mémoires culinaires) et entrepris par un lecteur complice, est construit comme une célébration des traditions et met l'accent sur ce nouvel enracinement.

Dans les mémoires culinaires, la nourriture et ses traditions incarnent le terroir, les coutumes et l'identité qui, ensemble, fournissent un point d'ancrage. Le récit autobiographique culinaire relate des souvenirs qui évoquent le monde authentique de l'enfance du narrateur, grâce à la nourriture et aux traditions familiales's ${ }^{2}$. Les fragments sélectionnés d'histoires personnelles, entrecoupés de recettes, déploient un récit autobiographique romancé. Imprégnées du "réalisme magique " qu'évoque Salman Rushdie (cité par Gonzalez, 2003 : 201) mêlant des éléments magiques ou oniriques à un contexte culturel vraisemblable, ces traditions culinaires ont, pour le narrateur, un pouvoir apaisant, transmis de manière mythique lorsque ces traditions sont respectées.

5 Bien qu'ils relatent avant tout des voyages endotiques (Viviès, 2003: 7), les mémoires culinaires peuvent également retracer des voyages exotiques amenant le lecteur vers le cœur même des cultures locales, à travers la description de nouveaux pays et de nouvelles traditions culinaires. Le narrateur ne cherche pas à constituer un texte référentiel tel que décrit par Christine Montalbetti. Des aperçus fugaces de paysages lointains sont fournis par l'évocation, parfois mythique, de la nourriture richement décrite, qui brouille la limite entre la fiction et le réel qui est souvent le propre des récits de voyages (Montalbetti, 1997: 67). Nous retrouvons ainsi dans les mémoires culinaires la problématique d'appellation de genre que Matthew Graves cerne dans sa notion d'hybridité générique, où nous découvrons un mélange d'autobiographie, de document référentiel, de fiction et de mémoire (Graves, 2006 : 17). Ces mémoires deviennent des récits de voyage revu par le prisme des souvenirs des traditions culinaires et leur hybridité générique permet de créer un passage initiatique au sein d'une quête d'identité.

Cette hybridité souligne la littérarité des textes: les mémoires culinaires édifient des mythes qui nous racontent des histoires de quête d'origines. La représentation du réel est choisie et ajustée en fonction de l'histoire racontée, ce que Boris Cyrulnik appelle le « remaniement imaginaire» de l'autobiographie (Cyrulnik, $2014: 168$ ). Dans ce contexte, l'évocation des traditions culinaires investit la nourriture d'une dimension miraculeuse qui permet aux narrateurs de ces mémoires d'accéder à une liberté fondée sur des rites culturels et non à l'écart des traditions. L'évasion vers un ailleurs idéalisé, suggère Guignery, n'est qu'un subterfuge pour effectuer un retour sur soi et s'interroger sur sa propre identité (Guignery, 2006: 38). Ainsi, comme le disait Darley dans Justine de Lawrence Durrell, «[t]his is no journey for the feet [...]. Look into yourself, withdraw into yourself and look» (Durrell, 2012 : 148). 
Ces mémoires tissent des récits de longs périples. La remémoration des souvenirs est intensifiée par la distance géographique, culturelle et spirituelle des évènements situés sur un continent lointain, et par une écriture empreinte d'un discours diasporique. Les souvenirs deviennent vitaux, même s'ils sont teintés de nostalgie ${ }^{3}$. Cette perspective insuffle à la littérature diasporique une dimension dichotomique, entre l'attirance du pays natal, le vieux monde, le berceau de l'identité (dans le cas des œuvres citées ici ${ }^{4}$ et la majorité des mémoires culinaires), et le nouveau monde dans lequel le narrateur doit s'intégrer.

Shoba Narayan, dans son mémoire Monsoon Diary (2003), a quitté son pays natal non pas à cause d'évènements dramatiques, mais parce que le monde a évolué. Sa génération doit suivre cette évolution naturelle de la société pour cultiver et protéger la force vitale de la remémoration "nostalgique » de sa communauté. La mutation de Narayan est rendue possible par l'acceptation d'une certaine autodétermination, qui suit les fluctuations de la vie pour résister au fatalisme, inhérente à sa culture, et qui pourrait déterminer son destin. Son récit postmoderne reflète un besoin de chercher une échappatoire et de suivre le mouvement socio-culturel. La diaspora, qui est a l'origine du discours de Narayan, doit reconnaître « a conception of identity which lives with and through, not despite, difference; by hybridity. Diaspora identities are those which are constantly producing and reproducing themselves anew, through transformation and difference» (Vertovec, $1997: 20)^{5}$. Ainsi le voyage emblématique que représente le "déplacement» devient un rite de passage et le récit de ce voyage assume momentanément le statut de Bildungsroman de par sa structure narrative.

Rudiger Kunow parle de la nourriture comme « a Janus-faced signifier pointing to the here of the uprooted diasporic life and the there of the homeland that they cannot go back to ${ }^{6}$ (Kunow, 2003: 173). Les mémoires culinaires permettent de construire des points d'ancrage d'identités imaginaires qui atténuent les effets du déracinement. Selon Barbara Waxman, les narrateurs diasporiques créent des espaces favorables à des échanges interculturels entre deux mondes. Ce faisant, ils détournent l'anxiété générée par l'incapacité potentielle à recréer leurs traditions de manière authentique dans leur pays d'adoption (Waxman, $2008: 366$ ).

10 Monsoon Diary (2003) est l'histoire d'une enfance passée en Inde, d'une adaptation culturelle au changement social et à la société américaine. Le livre décrit la formation de l'identité de la narratrice, façonnée par la nourriture, qu'elle célèbre dans ses souvenirs. En effet, la première statue que Narayan crée en tant qu'étudiante de sculpture aux ÉtatsUnis est celle d'une femme "rooted in the earth and reaching for the stars " (Narayan, 2003: 134), symbole de l'auteur enracinée dans ses traditions indiennes et tendant les mains vers un ailleurs.

11 Les maisons et les cuisines abritent les lieux d'enracinement où est mijotée l'identité individuelle et collective. Narayan affirme «I am from Kerala which means that regardless of where I live at any other point in my life, I will love coconuts in any form » (2003: 80). Dans son mémoire culinaire, Tender at the Bone (1998), la critique gastronomique Ruth Reichl encourage ses lecteurs à trouver, comme elle, un lieu où ils se sentent "grounded, fully there » (Reichl, $1998: 233)$. Narayan écrit également : « [w] hile the foreign flavours teased my palate, I needed Indian food to ground me " $2003: 118)$. "Grounded » a, bien entendu, un double sens en anglais, signifiant à la fois rétention et fondation. Narayan se montre attachée à sa famille et à sa culture qui lui donnent, selon son récit culinaire, une fondation solide à partir de laquelle elle peut prendre son envol. Son récit est ponctué de 
moments clés de sa vie où la cuisine lui permet de déplacer les frontières et de créer un passage, tout en respectant les traditions, le tout à travers des épreuves qui fournissent à Narayan les clés de son émancipation. L'ancrage dans ses traditions lui permet d'utiliser ces dernières pour accéder à une liberté qui lui serait autrement interdite. Narayan décrit comment les pratiques culinaires de sa région lui permettent non seulement de vivre son identité ethnique dans son pays d'accueil mais aussi de négocier son chemin dans le contexte de son déracinement. Les traditions culinaires permettent le "passage » d'une vie vers une autre, sans que l'on perde la première de vue.

Le poids de l'attache familiale dans la religion hindoue des $\operatorname{TamBram}^{7}$ se fait sentir. Tout comme la matérialisation symbolique de ce poids, la cérémonie thula bharam que les parents de Narayan célèbrent au temple nécessite qu'ils soient pesés avec un contrepoids de nourriture, pour ensuite payer leur poids en pièces et se débarrasser de leur péchés (Narayan, $2003: 4$ ). Les traditions culinaires, qui se trouvent au cœur de chaque chapitre, sont néanmoins une force libératrice ainsi qu'un artifice narratif à double tranchant. D'abord, ces traditions sont le sujet des défis qui créent une tension narrative au cours du récit. Elles sont également la matière première des épreuves que Narayan entreprend. L'héroïne doit cuisiner pour obtenir sa liberté. La mise à l'épreuve des traditions culinaires sert à les investir d'un pouvoir qui renforce la place émancipatrice que joue la nourriture dans le récit.

\section{Recette et anamnèse}

Dans Monsoon Diary, chaque recette a son propre contexte anecdotique, une légende traditionnelle ou bien une vignette familiale qui véhicule l'importance de la nourriture et annonce les pouvoirs quasi-magiques de ses plats et de leurs ingrédients. La recette de vatral kuzhambu est précédée d'un conte qui décrit ses qualités aphrodisiaques ; inji curry, ou pickles au gingembre et tamarin, commence par «the story goes like this " (Narayan, 2003 : 95); le curry à l'okra est introduit par la légende d'un pauvre homme qui, après l'avoir mangé, gagne aux échecs pour obtenir le droit de se marier avec la princesse. Les recettes sont aussi des offrandes de bienveillance, notamment le rasam, l'équivalent indien de la mythique soupe au poulet des Américains, aux légendaires propriétés réconfortantes et apaisantes (Narayan, $2003: 19$ ).

En analysant la fonction des recettes dans les mémoires, certains critiques estiment que la lecture des ingrédients et de leurs anecdotes suffit à elle seule à rendre l'évocation convaincante (Torreiro Pazo, 2014: 145). Pourtant, la formule récurrente des recettes, associées à une expérience sensorielle par la pratique, donne corps aux souvenirs, au sens réel, et sert à bâtir efficacement des bases d'identité stables et réparatrices. Évoquant le mémoire culinaire de Diana Abu-Jaber, The Language of Baklava (2004), contemporain de celui de Narayan, Carol Bardenstein explique : «[The] gesture that aims to restore the (past) whole through partaking of a (present) fragment [...] seems to heal and remove the previous tensions of displacement, or of being 'of two worlds.' » (2010 : 161)

Les recettes permettent d'éveiller les souvenirs sensoriels du passé, mettant des mots sur les sensations gustatives et olfactives. D'après David Sutton, le goût est un savoir incarné, une compétence apprise à travers une expérience sensorielle (Sutton, 2013) ${ }^{8}$. La localisation spatiale des souvenirs devient insignifiante et l'intention du récit de voyage de nommer les lieux pour en garder le souvenir est remplacée par une démarche plus 
intime. Les souvenirs, discrètement identifiés dans l'évocation d'un paysage sensoriel et initiatique, fournissent l'attache traditionnellement réservée aux lieux. Le récit décrit un voyage intérieur qui met en évidence la force formatrice des traditions.

La fonction de la nourriture peut donc être perçue comme à la fois « unifying and divisive, sharing cultures or drawing boundaries» (Waxman, 2010 : 366). Les recettes ancestrales des narratrices, associées à l'invitation du lecteur à la cuisine et au partage des secrets culinaires, peuvent soulager l'angoisse qui est au cœur de chaque histoire de déracinement. Dans la litanie des festins gourmands racontés dans Monsoon Diary, certaines cérémonies ont cette signification solennelle, comme Shraadham, une journée entière dédiée à l'hommage aux ancêtres, avec un repas destiné à nourrir les prêtres brahmanes: «it was a circle that defined my identity, my lineage and my place in the world» (Narayan, 2003: 90). Cette croyance permettait à Narayan et aux autres enfants de la famille d'attendre sereinement la fin du repas pour manger les quelques miettes que laissaient les prêtres.

\section{Les récits comme contes moraux}

17 Bâtie sur des tels récits de famille mystiques et mythiques, l'histoire de Narayan est construite comme un conte de fée, dans lequel la nourriture et ses traditions sont investies de pouvoirs spéciaux. Narayan accepte dans un premier temps, et par la suite s'impose à elle-même, une épreuve, montrant que dans son pays elle accepte de se laisser guider par sa famille, avant de se lancer un défi sur sa terre d'adoption, en Amérique. Dans les deux cas, son avenir dépend de son succès; la nourriture contrôle alors son destin.

Par l'épreuve que propose sa famille, qui consiste à cuisiner un repas qui doit recevoir son entière approbation, elle tente d'obtenir son accord pour partir faire ses études en Amérique. Ce repas sert symboliquement à prouver à sa famille que leurs traditions sont suffisamment ancrées pour que Narayan ne risque pas de "se perdre » dans une nouvelle culture $^{9}:$ « $[U]$ sing tattered family recipes, my mother's early instructions and gigantic cookbooks as guides, I began [...] the litany I learned at my mother's knee in my head [...] $»(2003: 106-107)$. Fidèle au dénouement des contes, l'héroïne réussit l'épreuve et obtient son billet pour partir vers la terre promise, synonyme de liberté.

19 En Amérique, la narratrice est libre de définir sa propre épreuve, cette fois-ci afin de pouvoir y rester. Manquant d'argent pour poursuivre ses études, elle décide de préparer un repas pour récolter des fonds, en demandant une participation aux invités. Cependant, en décidant de renoncer à sa cuisine traditionnelle pour préparer un repas éclectique de world cuisine, elle trahit ses origines et échoue à l'épreuve, réellement et symboliquement. Elle sauve le repas in extremis, qui s'avère immangeable pour la plupart des invités, en préparant upma, un plat indien simple et réconfortant: "as simple and comforting as a casserole, not to mention wholesome, quick to make and easy to like [...] ». Elle conclut : «[t]he whole thing was all right » (Narayan, 2003 : 145). Lorsqu'elle échoue à ses examens, Narayan se tourne vers la cuisine pour y trouver du réconfort: "I had always cooked to gain something: permission to go to America, a chance to stay an extra year, for money. In Memphis I cooked for what I had lost.» (2003: 161) Plus tard, son mariage trouvera également un équilibre harmonieux, grâce aux plats traditionnels qu'elle prépare pour son mari. 
20 Dans un contexte social diffèrent, mais aussi contraignant pour les femmes, du milieu du vingtième siècle, l'écrivaine américaine Mary Frances Kennedy Fisher décrit dans ses mémoires, centrés sur la nourriture et sa consommation, The Gastronomique Me (1989), comment elle a fui son mariage et son avenir dont la fin heureuse (bien qu'insatisfaisante pour elle) avait été prédestinée, pour rompre avec les traditions qu'elle estimait oppressantes. Elle invente une autre forme d'ancrage personnel qui, en même temps, empêche ses contemporains de cerner sa personne. En tant que femme, elle résiste à toutes les étiquettes: elle n'est plus épouse, et en tant qu'écrivaine, ses écrits ne se définissent pas comme des récits de voyage, ni comme des livres de cuisine; ils sont plutôt des essais qui distillent l'essence des deux.

21 Les mémoires culinaires, majoritairement d'auteurs femmes, permettent à ces écrivaines de trouver une place dans une société postcoloniale. La mission non-explicite de ces femmes mémorialistes est de réconcilier deux cultures et de faciliter l'évolution de leurs traditions. D'après Al-Hassan Golley, les femmes ne se reconnaissent plus dans les représentations culturelles et doivent développer une conscience parallèle, exprimée dans des métaphores d'oppression et de libération qui rythment Monsoon Diary (Al-Hassan Golley, 2003 : xv). Dans les mémoires culinaires, les traditions servent autant d'ancrage que de passage vers une liberté de choix. Ces auteures exploitent ce qu'Anita Mannur nomme "culinary citizenship ": "that which grants subjects the ability to claim and inhabit certain identitarian positions via their relationship to food» (Mannur , $2010: 29)^{10}$.

Les textes articulent les expériences de personnes et de groupes marginalisés à travers le vécu personnel (Anderson, 2001: 97). Narayan agit pour sa communauté et pour des femmes indiennes qui n'ont pas la liberté des Américaines. Elle vient d'une société «[...] where women deferred to men in public but ruled the roof in private ». Sa mère était " tethered by rules and tradition, and limited by her own vision of herself " (Narayan, 2003 : 124). Le narrateur autodiégétique est sensible aux changements : "India's fatalism was in direct contrast to the flux I felt in America. Everyone was moving, searching, asking for more." (Narayan, 2003 : 124) Elle témoigne du fait que malgré la désapprobation de sa famille pour la carrière choisie par son frère dans la marine marchande, toute la famille était présente pour lui faire ses adieux à son départ au collège, démonstration de leur bénédiction tacite (103).

Les autobiographies postmodernes, qui reposent sur « l'absolue relativité de tout ce qui passe pour réel, ce qui a pour conséquence l'abolition de la frontière entre réalité et fiction » (Toro, $2004:$ 3) permettent d'imaginer une vie multidimensionnelle (Jelinek, 1986: 134). Les mémoires culinaires sont dans des récits fragmentés, marqués par l'hybridité et les notions de mobilité et de traversée (Anderson, 2011 : 107). Estelle Jelinek indique que les récits disjonctifs, aux formes discontinues, reflètent, de manière symboliquement appropriée, les facettes fragmentées et multidimensionnelles des vies de femmes (Jelinek, 1986: 188). Fisher (1989), par sa réaction à la société en mutation pendant et après la guerre, se marginalise, brouillant les genres littéraires de ses écrits et refusant de se plier aux normes sociétales. Elle est fière d'avoir assouvi la faim de ses invités et en même temps d'avoir réveillé leurs désirs enfouis, lors d'un repas peu conventionnel de par son menu et son service informel. 


\section{Le point d'ancrage dans un nouveau monde}

24 qu'héritées. Ses voyages « diasporiques » sont, en fait, un " retour » choisi. Elle est attirée par le vieux continent, fixant comme point d'amarrage les traditions culinaires francoméditerranéennes. Ces traditions lui permettent d'assurer sa liberté, par rapport à ses propres traditions, dans un monde hostile aux femmes indépendantes. Elle est ainsi capable de s'enrichir affectivement, créant un ancrage dans les connaissances et les plaisirs du palais. "I saw food as something beautiful to be shared with people instead of a thricedaily necessity. » Sa narration, comme sa nourriture, est généreuse : «I with my brain and my hands have nourished my beloved few, [...] I have concocted a stew or a story, a rarity or a plain dish, to sustain them truly against the hungers of the world. » (Fisher, 1989: 18) Préparer la nourriture pour apaiser la faim lui donne un pouvoir en tant que femme: "The stove, the bins, the cupboards, I had learned forever, make an inviolable throne. From them I ruled; temporarily I controlled. I felt powerful and I loved that feeling. " (Fisher, 1989: 18) Fisher relie la nourriture et la faim à un manque d'amour et de reconnaissance qu'elle estime être au cœur de chaque être, émanant des besoins existentiels de chacun et des crises sociales.

Fisher explique : "[to] be happy you must have taken the measure of your powers, tasted the fruits of your passions, and learned your place in the world. » (1989: XI) "The Measure of my Powers » est le titre de onze des vingt-six chapitres de ses mémoires dans lesquelles elle fait l'évaluation répétée de sa capacité d'agir face aux normes sociétales qui l'enferment. Ces mots ponctuent le récit comme un refrain, de la même manière que résonnent les recettes dans d'autres mémoires. Cinq des chapitres restants sont appelés «Sea Change » et décrivent chacun de ses voyages à travers l'océan, les ruptures qu'ils représentent, et l'évolution de la narratrice lors de ces semaines en mer où la nourriture et l'acte de manger deviennent son ancrage et assure son passage vers une liberté d'action. Innovatrice de changement social et soucieuse de le transmettre, Fisher partage son ancrage : ils goûteront, dit-elle, «[...] not only the solid honesty of my red borscht, but the new flavor of the changing world.» (1989: 102) Comme les recettes du livre de Shoba Narayan, Fisher cherche à mettre des noms sur les zones d'ombres pour consolider son expérience et ses souvenirs.

Fisher quitte son mari pour échapper à sa vie banale de «faculty wife » en Amérique. Elle lève l'ancre et prend la mer (littéralement) avec un autre homme, une nouvelle fois pour la France, après une première période de résidence dans ce pays, l'incarnation, pour elle, du symbole de liberté. Lors du voyage, elle fête sa fuite de son " private soap-opera » (1989: 130). Malgré son mépris pour les conventions sociales, elle se protège derrière sa " gastronomic liberty». Fisher voyage par moment seule aussi, l'océan la séparant de ses deux hommes, le bateau, l'ancre levée, devenu le lieu d'ultime liberté morale. Avec chaque voyage, en s'ancrant davantage dans les rituels et les plaisirs gastronomiques, par lequels elle s'auto-définit, Fisher découvre une liberté de mouvement plus ample :

And the eighth and ninth, the tenth, eleventh, twelfth trips? What have they do do with the gastronomical me? [...] My hungers altered: I knew better what and how to eat, just as I knew better how I loved other people and even why. $(1989: 180)$

Lors d'un des voyages, le regard des autres passagers la force à donner forme à sa stratégie pour s'en protéger : l'art de manger seule. Elle développe un comportement qui la garde éloignée de toute " magouille » (« aloof of skulduggery»), à l'écart de ces dangers

ILCEA, 28 | 2017 
moraux auxquels elle fait discrètement allusion (Fisher, 1989 : 182). Ainsi elle obtient une liberté et défend sa solitude simplement dans sa façon de dîner. Elle pouvait aussi choisir avec qui elle mangeait, "[...] but in general I preferred to eat by myself, slowly, voluptuously, and with an independence which hardened me against the coldness of my cabin and my thoughts [...] It has often saved me and my reason too [...]» (1989: 183). Son isolement lui donne le courage d'affronter les dangers du monde.

Hélène Cixous considère l'écriture féminine comme une façon de rétablir un rapport de jouissance dans le corps de la femme. On trouve cette vision utopique de la créativité féminine chez Fisher (Moi, 2002 : 119), qui éprouve une sorte de jouissance dans le plaisir sensuel de l'acte de manger. Le chapitre final intitulé "Feminine Ending " célèbre son succès à trouver sa liberté, comme elle le définit. Fisher agit seule en adoptant les traditions gastronomiques pour ses propres fins, tandis que pour Narayan, dont le corps subit la participation aux rites traditionnels, les traditions représentent les limites sociales de sa communauté. Elle doit régulièrement s'alimenter des plats traditionnels pour rester ancrée, "grounded », dans cette communauté (Torreiro Pazo, 2014 : 142).

Selon Anthony Cohen les rituels ont une place centrale «[...] in the répertoire of symbolic devices through which community boundaries are affirmed and reinforced [...]", augmentant pour le narrateur son sens d'appartenance (Torreiro Pazo, 2014 : 149). Narayan mange le riz parfumé au ghee, dans le rite initiatique de sa naissance, symbolisant son respect et son engagement vis-à-vis des valeurs et des traditions de sa communauté. Cependant le symbolisme est sans équivoque dans son rejet immédiat de ce mets. Cela laisse entendre que cette petite fille pourrait éventuellement rejeter les valeurs et les traditions de la communauté. Heureusement à la deuxième tentative elle mange ce qu'on lui propose avec appétit et sa mère tient à préciser que la première préparation n'était simplement pas bonne. Cela signifie que les traditions ne suffisent plus pour sa fille: la nouvelle génération y adhère à condition que cela soit un plaisir sensuel, gustatif et nourrissant.

Monsoon Diary s'achève sur le tableau harmonieux d'une famille indienne en paix avec ses traditions et sa vie actuelle. Le choix de la nourriture et les rituels s'accommodent au nouveau lieu, mais l'esprit de respect et de fidélité reste inchangé. Le repas de " Thanksgiving » que Narayan célèbre avec la famille en Floride est le signe d'une adaptation transculturelle réussie: la nourriture traditionnelle est préparée à côté des plats américains ${ }^{11}$. L'ancrage reste solide et pourtant désormais aligné avec une nouvelle liberté culturelle. Sans la fondation des traditions et des cultures, le repas n'aurait pas été possible: "We were a group of Indians gathering to give thanks to America for its bounty [...] ", bien que selon leur tradition, "[t]he eating began at dawn and didn't end until midnight " (Narayan, $2003: 216-8)^{12}$. Ces mémoires, qui nous offrent des récits de voyages culturels et spirituels, transforment le rôle des traditions, tout en les respectant comme autant de points d'ancrages qui forment, paradoxalement, une passerelle vers une nouvelle identité caractérisée par un trait d'émancipation culturelle. 


\section{BIBLIOGRAPHIE}

ABU-JABER Diana (2005), The Language of Baklava, New York : Random House.

AL-HASSAN GolLEy Nawar (2003), Reading Arab Women's Autobiographies: Shahrazad tells her story, Austin : University of Texas Press.

ANDERSON Linda (2011), Autobiography [2001], Londres : Routledge.

BARDENSTEIN Carol (2010), « Beyond Univocal Baklava: Deconstructing Food-as-Ethnicity and the Ideology of Homeland in Diana Abu Jaber's The Language of Baklava », Journal of Arabic Literature, 41(1/2), 160-179,<http://www.jstor.org/stable/20720609>.

BRYNE Eleanor (2008), « Passing through the Impasse. », M. Shackleton (dir.), Diasporic Literature and Theory. Where Now?, Newcastle upon Tyne : Cambridge Scholars Publishing.

ChAtelet Noëlle (2002), « Manger, c'est vivre », D. Lecourt \& P. Fedida (dir.), Manger pour vivre?, Paris : Presses Universitaires de France.

CYRULNIK Boris (2014), Sauve-toi, la vie t'appelle, Paris : Odile Jacob.

DURRELL Lawrence (2012), The Alexandria Quartet [1962], Londres : Faber and Faber.

FISHER M. F. K. (1989), The Gastronomical Me [1943], New York : Duell, Sloan and Pearce.

GonZALEz Madalena (2003), « Le Sourire du Jaguar : fiction ou propagande ? Rushdie à Rebours », J. Viviès (dir.), Lignes de Fuite : Littérature de Voyage du Monde Anglophone, Aix-en-Provence : Université de Provence.

GRAVES Matthew (2006), « Le renouveau du récit de voyage : du New Journalism au New Travel Writing ", J. Viviès (dir.), Récits de Voyage et Romans Voyageurs, Aix-en-Provence : Université de Provence.

GUIGNERY Vanessa (2006), « Voyage au cœur du voyage : The Art of Travel (2002) d'Alain de Botton », J. Viviès (dir.), Récits de Voyage et Romans Voyageurs, Aix-en-Provence : Université de Provence.

JELINEK Estelle C. (1986), The Tradition of Women's Autobiography: From Antiquity to the Present, Massachusetts : Twayne Publishers.

KeLLER Isabelle (2002), « Prospero's Cell and Bitter Lemons. Aux frontières de la fiction et du récit de vie : les îles de la création ", J. Viviès (dir.), Lignes d'Horizon: Récit de voyage de la littérature anglaise, Aix-en-Provence : Université de Provence.

KunOw Rudiger (2003), « Eating Indian(s): Food, Representation, and the Indian Diaspora in the United States », T. Döring, M. Heide, S. Mühleisen \& C. Heidelberg (dir.), Eating Cultures: The Poetics and Politics of Food, Heidelberg : Winter, 151-75.

MANnUR Anita (2002), Culinary Fictions: Food in South Asian Diasporic Culture, Philadelphie : Temple University Press.

MoI Toril (2002), Sexual/Textual Politics [1985], Londres : Methuen.

MonTAlBetTi Christine (1997), Le Voyage, Le Monde et la Bibliothèque, Paris : Presses Universitaires de France. 
NARAYAN Shoba (2003), Monsoon Diary, New York : Random House.

OLNEY James (1973), Metaphors of Self: The Meaning of Autobiography, Princeton : Princeton University Press.

REICHL Ruth (1998), Tender at the Bone, Growing Up at the Table, New York : Random House.

RUSHDIE Salman (1991), Imaginary Homelands, Londres : Granta Books.

SRINIVAS Tulasi (2013), « 'As Mother Made it': The Cosmopolitan Indian Family, 'Authentic' Food, and the Construction of Cultural Utopia », C. Counihan \& P. Van Esterik (dir.), Food and Culture: A Reader, Londres, New York : Routledge, 355-376.

SutTon David (2013), " Cooking Skills, the Senses, and Memory: the Fate of Practical Knowledge », C. Counihan \& P. Van Esterik (dir.), Food and Culture: A Reader, Londres, New York : Routledge, 299-320.

ToRo Alfonso de (2004), « La "nouvelle autobiographie” postmoderne ou l'impossibilité d'une histoire à la première personne : Robbe-Grillet, Le miroir qui revient, et de Doubrovsky, Livre brisé ", A. de Toro \& C. Gronemann (dir.), Autobiographie Revisited: Theorie und Praxis neuer autobiographischer Diskurse in der französischen, spanischen und lateinamerikanischen Literatur, Hildesheim/Zürich/New York : Olms, 79-113.

TORREIRo PAZo Paula (2014), Diasporic Tastescapes: Intersections of Food and Identity in Asian American Literature (thèse de doctorat), Université de la Corogne, Espagne.

VERTOVEC Steven (1997), « Three meanings of 'diaspora', exemplified among South Asian religions ", Diaspora: A Journal of Transnational Studies, 6.3, 277-299.

VIVIES Jean [dir.] (2003), Lignes de Fuite : Littérature de Voyage du Monde Anglophone, Aix-enProvence : Université de Provence.

WAUGH Patricia (1989), Feminine Fictions: Revisiting the Postmodern, Londres : Routledge.

WAXMAN Barbara Frey (2008), « Food memoirs: What they are, why they are popular, and why they belong in the literature classroom », College English, 70(4), 363-383.

\section{ANNEXES}

Virginia Allen-Terry Sherman est doctorante à l'Institut des Langues et Cultures d'Europe, Amérique, Afrique, Asie et Australie (ILCEA4), Université Grenoble-Alpes, sous la direction du Professeur Catherine Delmas. Sa thèse est intitulée « Diaspora and displacement: the evocation of traditions, origins and identity in culinary memoirs, an emerging genre ». Sa recherche se focalise sur les mémoires culinaires contemporains, la place de la mémoire et la construction de l'identité dans les textes autobiographiques, et les mémoires culinaires comme forme de récits de voyages émergent. Publication récente : Allen-Terry Sherman, Virginia, Cartellier-Veuillen, Éléonore, Dalrymple, James, Fruoco, Jonathan (dir.), (Re)writing and Remembering: Memory as Artefact and Artifice, Newcastle upon Tyne : Cambridge Scholars Publishing, 2016. 


\section{NOTES}

1. Dans la diversité du genre de récit «culinaire ", nous pouvons constater une dichotomie entre ce que Viviès évoque comme le voyage endotique (où le protagoniste part à la recherche de luimême) et le voyage exotique (où le protagoniste cherche l'autre) [Viviès, 2003].

2. Cependant la nostalgie peut masquer, pour le lecteur et le narrateur, la complexité des actes de remémoration. Ces récits nostaligiques, « Nostalgically framed narratives", doivent être aussi perçus, d'après Anita Mannur (2010), comme des métacritiques portant sur la canalisation des souvenirs du passé à travers le rapport qu'on peut avoir avec les pratiques culinaires, ostensiblement immuables, et ses conséquences.

3. D'après Anita Mannur, la nostalgie qui relie les immigrés à leur passé est pré- voire surdéterminée: "the desire to remember home by fondly re-creating culinary memories cannot be understood merely as a reflectively nostalgic gesture; rather such commemorative acts must be read as a commentary on what it means to inhabit different diasporic locations while constantly battling the implications of routing memory and nostalgia through one's relationship to culinary practices » (Mannur, $2010: 20)$.

4. M. F. K. Fisher (1989), The Gastronomical Me et Shoba Narayan (2003), Monsoon Diary.

5. Vertovec cite Stuart Hall.

6. Je souligne.

7. «Tamil Brahmin », Indiens Tamoul de caste Brahmane du sud-est de l'Inde.

8. Sutton parle notamment de la préparation de la nourriture comme compétence acquise à travers la mémoire et les sens (300) et précise que les recettes «[...] act more as memory-jogs for previous learnings that have been acquired through experience » (Sutton, 2013 : 303).

9. Tulasi Srinivas explore une évolution dans la consommation sociétale en associant la construction de l'identité ethnique indienne et l'anxiété autour de la consommation authentique avec l'émergence des plats indiens préemballés: "Food consumption is seen as a "narrative of affiliative desire" that affectively recreates caste, micro regional and other social identity groupings for the cosmopolitan Indian family. Fueled by a "narrative of anxiety" over "authentic" foods-"as mother made them"-the act of eating is transformed into a performance of "gastro-nostalgia" that attempts to create a cultural utopia of ethnic Indian-ness that is conceptually de-linked from the Indian nation state. » (Srinivas, $2013: 356$ )

10. Mannur estime que les réinterprétations des modèles d'identité nationale officielles et traditionnelles des femmes de l'Asie du Sud sont souvent définies par un idiome culinaire (Mannur, $2010: 29$ ).

11. Bien qu'idéalisé, ce récit contredit le propos de Mannur pour qui la nostalgie vestigiale pour le «homeland » peut générer une sorte de fiction culinaire où les traditions culinaires restent « discrete, immutable and coherent expressions of unfaltering national essence » (Mannur, $2010: 29$ ).

12. D'après Mannur, Thanksgiving a la force d'un symbolisme particulièrement pertinent dans l'exploration des passages culturels : "Thanksgiving is a particularly appropriate site from which to imagine a form of safe multiculturalism. » (Mannur, 2010 : 166) 


\section{RÉSUMÉS}

Les traditions culinaires, évoquées dans les récits autobiographiques, sont présentées comme vecteur d'une force qui, paradoxalement, permet aux narrateurs d'obtenir un degré de liberté au sein des contraintes qu'impose leur culture, tout en restant ancrés dans leur identité culturelle. Les narrateurs tissent des récits imaginaires dans lesquels ils reconstruisent leur intégrité culturelle malgré un contexte diasporique. La dimension viatique de ces récits permet d'explorer des moments de "passage » où l'hybridité, emblématique du genre autobiographique culinaire, laisse une place à la différence. La nourriture et ses recettes apportent cohésion et fragmentation, caractéristiques de l'hybridité tant sur le plan culturel que sur le plan poétique. Comme autant d'éclats de miroir, elles permettent, à travers les sens, de rappeler ou de reconstruire des souvenirs des patries imaginaires évoquées par Rushdie (1991: 12), éloignés par le déplacement ou l'exil.

Culinary traditions, as evoked in autobiographical narratives, are presented as the agents of a force that, paradoxically, allows narrators to obtain a degree of liberty within the constraints of their culture, while, nonetheless, remaining anchored within their cultural identity. Narrators weave a narrative of imaginary dimensions in which they are able to reconstruct their cultural integrity despite a diasporic context. The travel perspective of these stories allows the author to explore moments of "passage", where hybridity, emblematic of this culinary autobiographic genre, makes room for difference. Food and its recipes bring, at once, both cohesion and fragmentation, inherent facets of hybridity, as much on a cultural as on a poetic level. Like the shards of a mirror, they make it possible, through the senses, to remember or re-member memories from what Rushdie calls "Imaginary Homelands" (1991: 12), distanced by displacement or exile.

\section{INDEX}

Mots-clés : littérature anglophone contemporaine, autobiographie, mémoire, auteurs femmes, récits de voyage, récits diasporiques, traditions culinaires, quête d'identité

Keywords : contemporary anglophone literature, autobiography, memoirs, women writers, travel writing, diasporic narratives, culinary traditions, identity search

\section{AUTEUR}

VIRGINIA ALLEN-TERRY SHERMAN

Doctorante à l'ILCEA4 